\title{
ANN Circuit Application of Complementary Resistive Switches
}

\author{
E. UÇAR, E. KARAKULAK and R. MUTLU
}

\begin{abstract}
Artificial neural networks are successfully used for classification, prediction, estimation, modeling and system control. However, artificial neural networks integrated circuits are expensive and not matured enough. Memristors or memristive systems which show a nonvolatile memory behavior has a high potential for use in artificial neural network circuit applications. Some memristive synapse or memristive neural network applications already exist in literature. The complementary memristor or resistive switch memories have been suggested as an alternative to one-cell memristor memories. Their sensing is more difficult and complex than the others. The complementary memristor memory topologies with a sensing node are also inspected in literature. To the best of our knowledge, a neural network circuit which is based on the complementary resistive switches with a sensing/writing node does not exist in literature yet. In this paper, several neural network circuits which are based on the complementary resistive switches with a sensing/writing node have been designed and examined for the first time in literature. Their analysis are given and simulations are performed to verify their operation. We expect that such a complementary resistive switch implementation may find use in artificial neural networks chips in the future.
\end{abstract}

Index Terms-Memristor, Memristive systems, Complementary resistive switches, Artificial neural networks, ANN circuits.

\section{INTRODUCTION}

$\mathrm{A}$ RTIFICIAL NeURAL Networks (ANN) are biologically inspired systems which are able to do classification, prediction, estimation, modeling and system control [1]. The neural networks integrated circuits are expensive and not commonly used $[2,3]$. If new neural networks integrated circuit topologies are found, this may help to cheapen their price and make them more common and rugged. One way to find such ANN topologies is to make use of new-found circuit elements. Memristor is such a new-found circuit element and it has a memory $[4,5]$.

ERDEM UÇAR, is with Department of Management Information Systems University of Trakya, Edirne, Turkey, (e-mail: erdemucar@trakya.edu.tr).

(D) https://orcid.org/0000-0002-8465-3396

ERTUĞRUL KARAKULAK, is with Department of Vocational School of Technical Sciences Namık Kemal University, Tekirdağ, Turkey, (e-mail: ekarakulak@nku.edu.tr).

\section{iD https://orcid.org/0000-0001-5937-2114}

REŞAT MUTLU, is with Department of Electronics and Communication Engineering Namık Kemal University, Çorlu, Tekirdağ, Turkey, (e-mail: rmutlu@nku.edu.tr).

\section{(iD https://orcid.org/0000-0003-0030-7136}

Manuscript received September 6, 2018; accepted January 18, 2019.

DOI: $\underline{10.17694 / \text { bajece. } 457902}$
It was predicted as the fourth missing circuit element by Leon Chua in 1971 [5] and has been declared found by a research team in 2008 [4]. Memristors or memristive systems which are systems behaving as memristors are under consideration for memory applications as well as analog applications. Two reviews on memristor and its memory applications can be found in [6,7]. Resistive switches are also declared as memristors by Chua [7]. The complementary resistive switches (CRS), which are anti-series connected memristors, are suggested as a way to decrease leakage current in resistive switches [8,9]. A new CRS topology with a sensing/reading line is suggested in [10].

Since memristor is a nonvolatile memory, it can also be used for making neural network circuits. Memristors or memristive circuit elements are able to provide artificial neural network circuits with adjustable gains required for training and a good performance since they have variable resistance values. Some studies on memristor-based artificial neural network circuits can be found in [11-13]. Recently, memristors have been used to create a chip that borrows design points from the brain and the prototype chip were able to recognize extremely simple black-and-white patterns [14]. A larger, more complex versions of the chip might make computers better at more difficult tasks such as understanding speech, images, and the world around them. That's why the new circuit element memristor is a promising candidate for artificial neural network circuits as well as the non-volatile memory domain with its high density and low power consumption. However, to the best of our knowledge, the complementary resistive switches or complimentary connected memristors have not been used to make a perceptron or an artificial neural network circuit in literature yet. In this paper, the CRS topology with the sensing line is used to make perceptron and ANN circuits. These CRS based perceptron topologies also make use of operational amplifiers for normalization and amplification purposes. The perceptron circuits given in literature do not have automatically adjustable negative or positive gains. Also for the first time in literature, the CRS based opamp amplifiers are able to give not only negative but also positive gains for the perceptron circuit. Then, it is shown how to use the CRS-based perceptrons to make a multi-layer artificial neural network circuit. The CRS model given in [15] is modified to model the CRS with a sensing line and it is used in simulations. A microcontrollerbased programmer which can be made using off-shelves components and a cheap microcontroller are suggested for programming such a perceptron or ANN circuit. 
The paper is arranged as follows. In the second section, a complementary connected resistive switches without and with a sensing line are briefly explained and the CRS model with nonlinear dopant drift given in [15] is modified for a sensing line. In the third section, first a Hopfield perceptron is reviewed, then various perceptron circuits are made using the CRS cells with sensing line, and as last it is shown how to make an ANN circuit based on the perceptron circuits. In the fourth section, a microprocessor-based programmer for the CRS-based perceptron is introduced and the programmer is simulated using Matlab $^{\mathrm{TM}}$. In the fifth section, the simulation results obtained using Matlab $^{\mathrm{TM}}$ for the CRS-based perceptron circuit is given. The paper is concluded with the sixth section.

\section{MODELING OF THE COMPLEMENTARY RESISTIVE SWITCHES}

\section{A. Complementary Resistive Switch Topology without and with a Sensing Line}

In this section, a CRS topology is to be explained briefly. CRS can be made of different materials $[16,17]$. A CRS made of PtGeSe-Cu-GeSe-Pt as in [10], materials is shown in Fig. 1. The CRS consists of a Platinum $(\mathrm{Pt})$ top electrode, an upper solid electrolyte (for instance, made of $\mathrm{GeSe}$ ), and an oxidizable middle electrode made of a material such as Copper $(\mathrm{Cu})$, a lower solid electrolyte, and a Platinum (Pt) bottom electrode. The equivalent circuit of the CRS can be modelled as a memristive element $U$ and a memristive element $\mathrm{L}$ connected in series with opposite polarities as also shown in Fig. 1. The merging copper electrode between the lower and the upper memristive elements. which is connected to outside is usable for writing, reading and sensing purposes of the CRS cells,

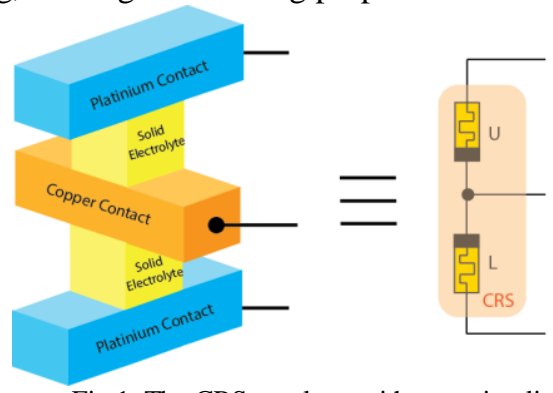

Fig.1. The CRS topology with a sensing line given in [10].

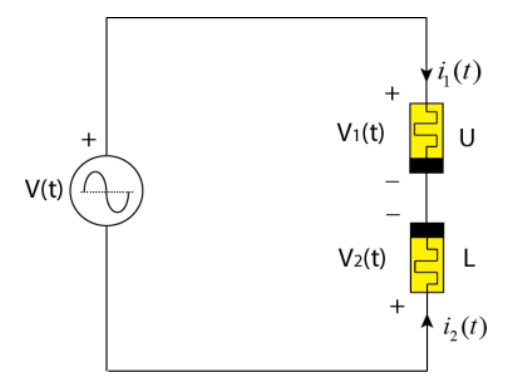

Fig. 2. The equivalent circuit of the CRS cell without a sensing line.

\section{B. Analytic Model of the Complementary Resistive Switches without a Sensing Line}

In this section, the CRS model given in [15] is briefly explained. The CRS model given in [15] is a nonlinear model and the resistance of a resistive switch in the CRS is dependent on the rate of change of the normalized dopant drift length, two threshold voltages and a two-variable window function. The equivalent circuit of the CRS cell without a sensing line is shown in Fig. 2. Considering the experimental data in [8], the rate of change of the state-variables are presented using two threshold voltages as the following,

$$
i_{1}(t)=-i_{2}(t)
$$

$$
V(t)=V_{1}(t)+V_{2}(t)=\left[R_{U}\left(x_{1}\right)+R_{L}\left(x_{2}\right)\right]-i_{2}(t)
$$

$$
\frac{d x_{1}}{d t}=\left\{\begin{array}{ccc}
\frac{\mu_{v} \cdot i_{1}(t) \cdot R_{o f f}}{D^{2}} f\left(x_{1}, i_{1}\right) & , & V_{T H 2} \leq V \\
0 & , & V_{T H 1} \leq V<V_{T H 2} \\
0 & , & -V_{T H 1}<V<V_{T H 1} \\
\frac{\mu_{v} \cdot i_{1}(t) \cdot R_{o f f}}{D^{2}} f\left(x_{1}, i_{1}\right) & , & V_{T H 2}<V \leq-V_{T H 1} \\
0 & , & V \leq-V_{T H 2}
\end{array}\right.
$$

$$
\frac{d x_{1}}{d t}=\left\{\begin{array}{ccc}
\frac{\mu_{v} \cdot i_{1}(t) \cdot R_{o f f}}{D^{2}} f\left(x_{1}, i_{1}\right) & , & V_{T H 2} \leq V \\
0 & , & V_{T H 1} \leq V<V_{T H 2} \\
0 & , & -V_{T H 1}<V<V_{T H 1} \\
\frac{\mu_{v} \cdot i_{1}(t) \cdot R_{o f f}}{D^{2}} f\left(x_{1}, i_{1}\right) & , & V_{T H 2}<V \leq-V_{T H 1} \\
0 & , & V \leq-V_{T H 2}
\end{array}\right.
$$

$\mathrm{x}_{1}=w_{1} / D$ and $\mathrm{x}_{2}=w_{2} / D$. Where $w_{1}$ and $w_{2}$ are oxidized length of resistive switches upper and lower respectively, $D$ is physical length of resistive switches. $\mathrm{V}_{\mathrm{TH} 1}$ and $\mathrm{V}_{\mathrm{TH} 2}$ are threshold voltages of upper and lower resistive switches. $\mu_{v}$ is mobility coefficient of oxygen ions, $i_{1}(t)$ and $i_{2}(t)$ are currents of resistive switches upper and lower respectively. $R_{\text {off }}$ is maximum resistance value, $\mathrm{R}_{\mathrm{ON}}$ minimum value of resistive element.

Simulink $^{\mathrm{TM}}$ toolbox of MATLAB ${ }^{\mathrm{TM}}$ is used for all the simulations in this paper. A voltage source of $V(t)=$ $V_{m} \cdot \cos (\omega t)=1,4 \cdot \cos (20000 \pi t)$ is used to excite the CRS. The CRS current, voltage and resistance (memristance) are given in Fig. 3 and its zero-crossing pinched hysteresis loop is shown in Fig. 4. 

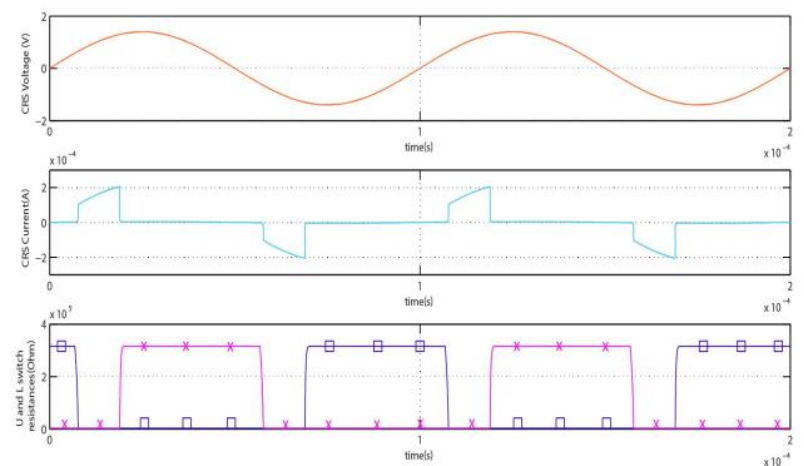

Fig. 3. The CRS current, voltage and memristances when excited with $V(t)=1,4 \cdot \cos (20000 \pi t)[15]$.

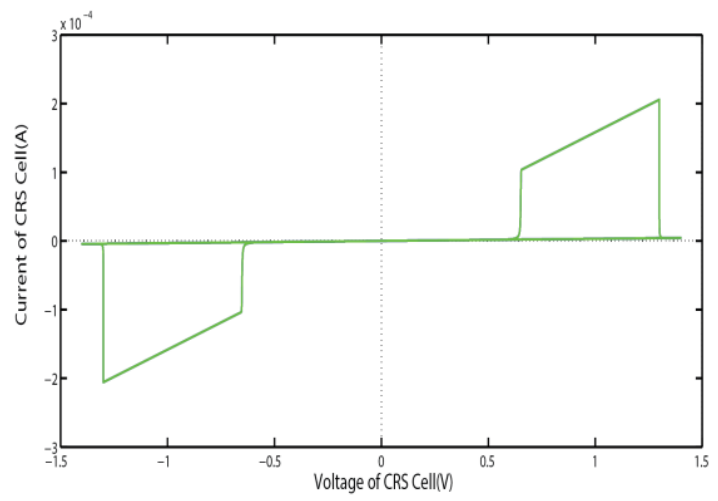

Fig. 4. The zero-crossing hysteresis loop of the CRS model when excited with $V(t)=V_{m} \cdot \cos (\omega t)=1,4 \cdot \cos (20000 \pi t)[15]$.

\section{Analytic Model of Complementary Resistive Switches with a Sensing Line}

In this section, the model of the CRS topology without a sensing line is modified for the CRS topology with a sensing line. The model of the CRS topology without a sensing line suggested in [8] is modified for the CRS topology with a sensing line. Its equivalent circuit can be obtained as shown in Fig. 5. It is same as the CRS circuit except the sensing line in Fig. 2 that makes the current of the resistive switches different than each other during writing and sensing processes.

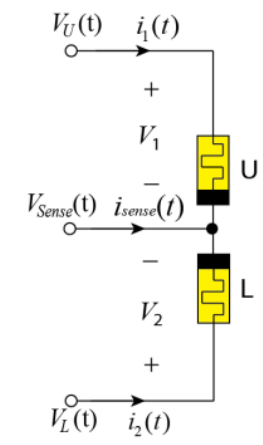

Fig. 5. The equivalent circuit of the CRS topology with a sensing line.

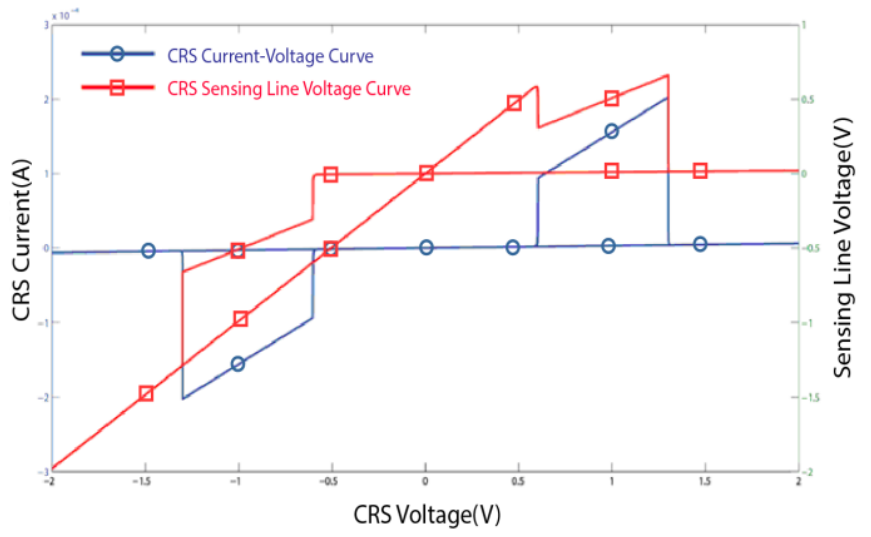

Fig. 6. Current-Voltage and sense voltage of CRS with sensing line.

In this section, the analytical model of the CRS topology with a sensing line is made. $V_{U}$ and $V_{L}$ are the node voltages shown in Fig. 5.

$$
\begin{aligned}
& V_{1}(t)=V_{U}-V_{\text {sense }} \\
& V_{2}(t)=V_{L}-V_{\text {sense }}
\end{aligned}
$$

In this case, the CRS cell currents are

$$
\begin{aligned}
& i_{1}(t)=\frac{V_{1}}{R_{1}}=\frac{V_{U}-V_{\text {sense }}}{R_{1}} \\
& i_{2}(t)=\frac{V_{2}}{R_{2}}=\frac{V_{L}-V_{\text {sense }}}{R_{2}}
\end{aligned}
$$

In this paper, it is chosen to keep the upper CRS switch at the minimum resistance value and only to tune the lower CRS switch resistance for the perceptron gains desired. If only the lower CRS switch is selected throughout the sense line and the lower terminal,

$$
V_{U}=V_{\text {sense }} \text { and } V_{1}(t)=0
$$

In this case, the current of the upper CRS element is

$$
i_{1}(t)=0
$$

In this paper, the upper CRS switch is hold at LRS state during circuit operation. Its purpose is to provide threshold and keep the lower CRS switch voltage is less than the threshold voltage 
to prevent its resistance drift. Therefore, the desired resistance or the memristance of the lower resistive switch is given as,

$$
R_{1}=R_{o n}
$$

This means the CRS upper switch resistance must be reset to $\mathrm{R}_{\mathrm{on}}$ at the beginning of the programming operation. A good CRS switch is needed to keep the memristance drift acceptable and perhaps this can be done using new materials in the future. During programming,

$$
i_{2}(t)=\frac{V_{\text {sense }}(t)}{R_{2}}
$$

During normal operation of the CRS based perceptron, the model given in the previous section can be used for the simulations, i.e.

$$
i(t)=i_{1}(t)=-i_{2}(t) \text { and } i_{\text {sense }}(t)=0
$$

\section{THE CRS-BASED PERCEPTRON MODEL}

\section{A. Hopfield Perceptron Circuit Model and Perceptron Circuit with Memristors}

A perceptron is a biologically inspired artificial neural network. Its block diagram is shown in Fig. 7.a. and the Hopfield perceptron circuit, which is the first perceptron circuit given in literature, is shown in Fig. 7.b [18,19]. It is made of a current source for the offset input, resistors, a capacitor and a nonlinear circuit element for the activation function. The following differential equation describes the Hopfield perceptron circuit:

$$
\frac{d u_{i}}{d t}=-\frac{u_{i}}{\tau_{i}}+\sum_{j=1}^{n} T_{i j} v_{j}+I_{i}
$$

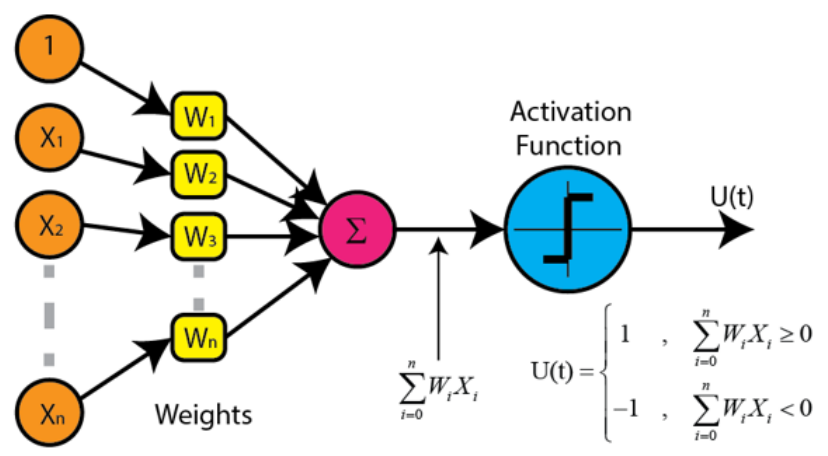

Inputs (a)

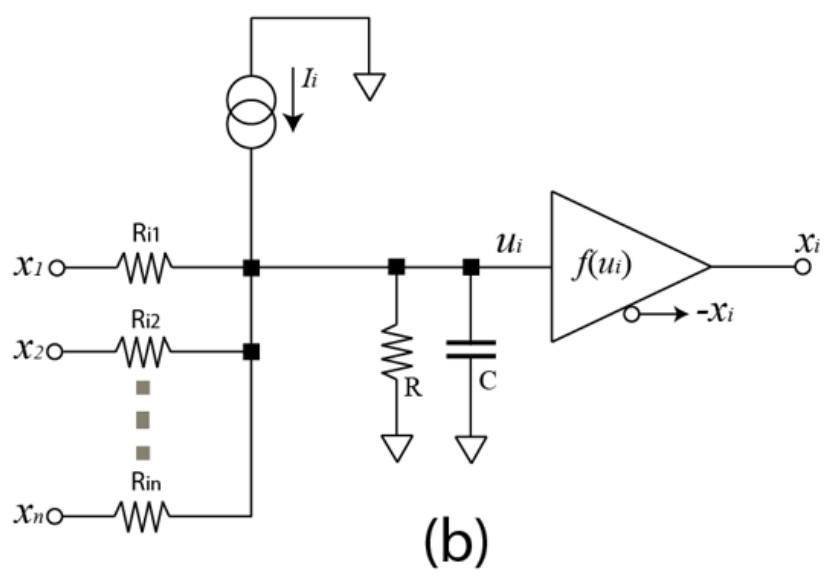

Fig. 7. b) The Hopfield Perceptron Circuit.

\section{B. The CRS-based Perceptron Topology and Its Model}

The circuit in Fig. 7.b has constant resistors and therefore for each perceptron application, different resistors must be mounted to tune the perceptron circuit. If the perceptron is trained again and the gains are changed, the resistors must be replaced. Using variable resistors or potentiometers instead resistors may solve the need to replace the gain resistors. Artificial Neural Network and CRS based perceptron block can be seen in Fig. 8. The CRS-based perceptron circuit is given in Fig. 9. It replaces the resistors with CRS switches. The CRS switches resistances or memristances can be adjustable easily using pulses [20,21]. The upper switches are kept at $R_{\text {on }}$ resistance value and the lower switches are tuned to have the necessary resistance value to provide the needed gains. The opposite method of keeping the lower switches at $R_{\text {on }}$ and tuning the upper switch resistances can also give the same performance. The input opamps are used for scaling the input voltages and to provide a voltage less than the threshold voltage.

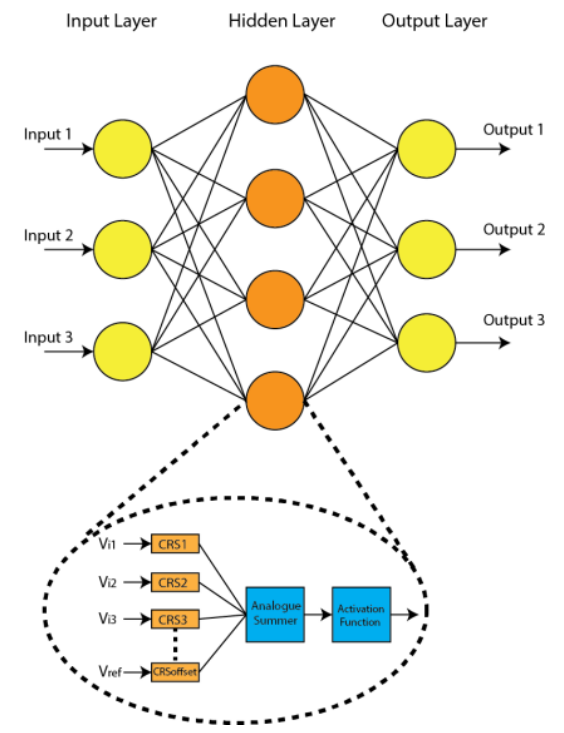

Fig. 8. Artificial Neural Network with CRS Block Diagram.

Fig. 7. a) Perceptron block diagram 


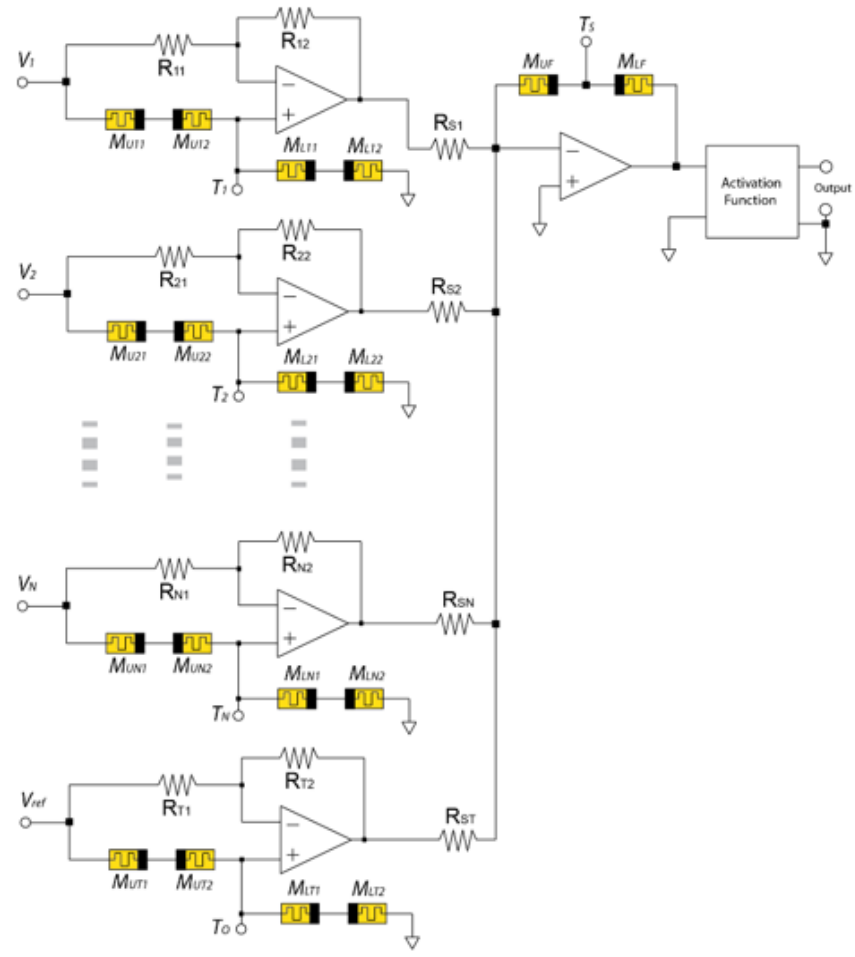

Fig. 9. The Proposed CRS-based Perceptron Circuits.

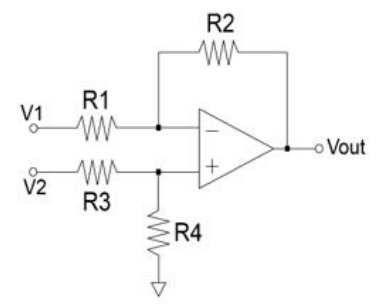

(a)

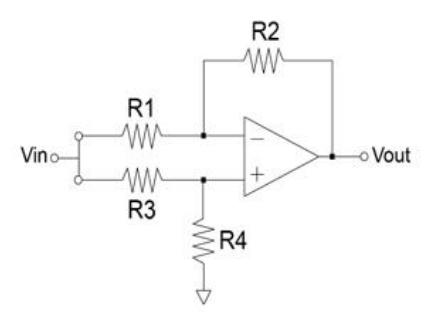

(b)

Fig. 10. a) Differential amplifier b) Differential amplifier for the negative gain or the negative weighting factors.

Perceptron gains may have positive or negative values. The same perceptron may require positive and negative gains at the same time for a given problem. Resistor or memristor based perceptron circuits given in literature have already had either positive or negative gains for weighting factors but not both for the same perceptron application and used dc offset. In this paper, also for the first time in literature, it is also shown how to obtain a weighting factor ranging from -1 to +1 . Using differential amplifiers and memristors to obtain not only negative but also positive weighting factors. An inverting amplifier has negative gain and a non-inverting amplifier has positive gain. A differential amplifier can be made to have either positive or a negative gain by adjusting its gains its resistors. Differential amplifiers are shown in fig. 10. Output voltage of the differential amplifier in Fig. 10 is,

$$
V_{\text {out }}=\left(1+\frac{R_{2}}{R_{1}}\right)\left(\frac{R_{4}}{R_{3}+R_{4}}\right) \cdot V_{2}-\left(\frac{R_{2}}{R_{1}}\right) \cdot V_{1}
$$

For $\mathrm{V}_{1}=\mathrm{V}_{2}$ (The inputs are connected together) and $\mathrm{R}_{1}=\mathrm{R}_{2}$, the gain of the amplifier in Fig. 10.b becomes,

$$
G=\left(\frac{R_{4}-R_{3}}{R_{4}+R_{3}}\right)
$$

Considering $\mathrm{R}_{4}>\mathrm{R}_{3}$, the gain becomes positive and vice versa. The amplifier is to be called negative-positive gain amplifier (NPGA) from now on. For the parametric analysis of the gain of negative-positive gain amplifier, a parameter which is the ratio of the resistance $\mathrm{R}_{4}$ to the resistance $\mathrm{R}_{3}$ is defined as $\gamma=$ $R_{4} / R_{3}$. Therefore, the gain can now be given as,

$$
G=\frac{\gamma-1}{\gamma+1}
$$

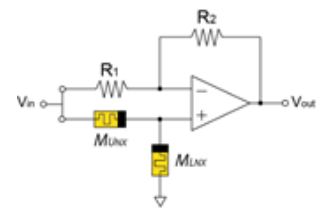

(a)

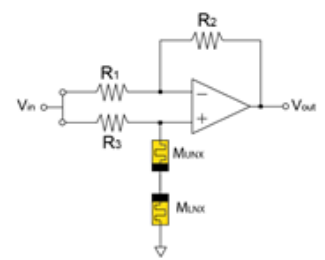

(d)

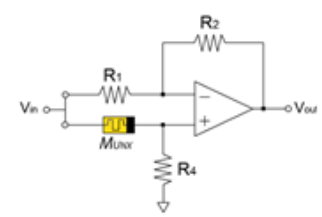

(c)

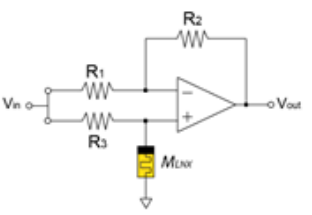

(b)

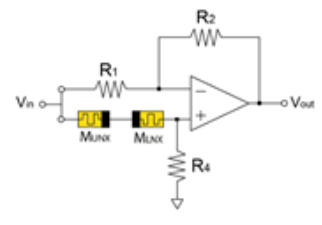

(e)

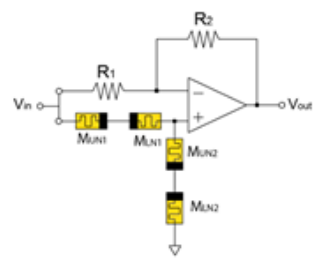

(f)
Fig. 11. The various negative-positive gain circuits a) The CRS based NPGA circuit b) The NPGA with a lower resistive switch c)The NPGA with an upper resistive switch d) The NPGA with a lower CRS e) The NPGA with an upper CRS f) The NPGA with both upper and lower CRSs. 
TABLE I GAIN EQUATIONS OF NEGATIVE POSITIVE GAIN AMPLIFIERS

\begin{tabular}{|c|c|c|}
\hline $\begin{array}{l}\text { Types of } \\
\text { Negative } \\
\text { Positive } \\
\text { Gain } \\
\text { amplifiers }\end{array}$ & Gain Equations & $\begin{array}{l}\text { Minimum } \\
\text { CRS } \\
\text { Threshold } \\
\text { Voltage }\end{array}$ \\
\hline $\begin{array}{l}\text { CRS } \\
\text { Based } \\
\text { Negative } \\
\text { Positive } \\
\text { Gain } \\
\text { Amplifier }\end{array}$ & $G=\frac{M_{L N X}-M_{U N X}}{M_{L N X}+M_{U N X}}$ & $\mathrm{~V}_{\text {TH1 }}$ \\
\hline $\begin{array}{l}\text { Lower } \\
\text { Resistive } \\
\text { Switch } \\
\text { Negative } \\
\text { Positive } \\
\text { Gain } \\
\text { Amplifier }\end{array}$ & $G=\frac{M_{L N X}-R_{3}}{M_{L N X}+R_{3}}$ & $\mathrm{~V}_{\text {TH1 }}$ \\
\hline $\begin{array}{l}\text { Upper } \\
\text { Resistive } \\
\text { Switch } \\
\text { Negative } \\
\text { Positive } \\
\text { Gain } \\
\text { Amplifier }\end{array}$ & $G=\frac{R_{4}-M_{U N X}}{R_{4}+M_{U N X}}$ & $\mathrm{~V}_{\text {TH1 }}$ \\
\hline $\begin{array}{l}\text { Lower } \\
\text { CRS } \\
\text { Negative } \\
\text { Positive } \\
\text { Gain } \\
\text { Amplifier }\end{array}$ & $G=\frac{\left(M_{L N X}+M_{U N X}\right)-R_{3}}{\left(M_{L N X}+M_{U N X}\right)+R_{3}}$ & $\mathrm{~V}_{\text {TH1 }}$ \\
\hline $\begin{array}{l}\text { Upper } \\
\text { CRS } \\
\text { Negative } \\
\text { Positive } \\
\text { Gain } \\
\text { Amplifier }\end{array}$ & $G=\frac{R_{4}-\left(M_{L N X}+M_{U N X}\right)}{R_{4}+\left(M_{L N X}+M_{U N X}\right)}$ & $\mathrm{V}_{\mathrm{TH} 1}$ \\
\hline $\begin{array}{l}\text { Upper } \\
\text { And } \\
\text { Lower } \\
\text { CRS } \\
\text { Negative } \\
\text { Positive } \\
\text { Gain } \\
\text { Amplifier }\end{array}$ & $\begin{array}{c}G \\
=\frac{\left(M_{L N X 2}+M_{U N X 2}\right)-\left(M_{L N X 1}+M_{U N X 1}\right)}{\left(M_{L N X 2}+M_{U N X 2}\right)+\left(M_{L N X 1}+M_{U N X 1}\right)}\end{array}$ & $\begin{aligned} & \mathrm{V}_{\mathrm{TH} 2} \\
= & 2 \mathrm{~V}_{\mathrm{TH} 1}\end{aligned}$ \\
\hline
\end{tabular}

The resistance of the resistor or the minimum resistance value of the memristor which is connected between the positive input and the input voltage must be chosen. In this paper, we have chosen to calculate the resistance value as the geometric average of maximum and minimum values of memristive element. The resistance equations used for the calculations and the gain equations are given in Table I. All the proposed negative-positive gain amplifiers can be seen in Fig. 11. For the CRS model in [15], $\mathrm{R}_{\mathrm{ON}}=3,16.10^{3} \Omega$ and $\mathrm{R}_{\mathrm{OFF}}=316.10^{3} \Omega$. Using Eq. 15, 16 and the Eq. 17 given in Table 1, the gain curves of all negative-positive gain amplifiers are drawn and shown in Fig. 12.

$$
R_{\text {reg }}=\sqrt{M_{\max } \cdot M_{\min }}
$$
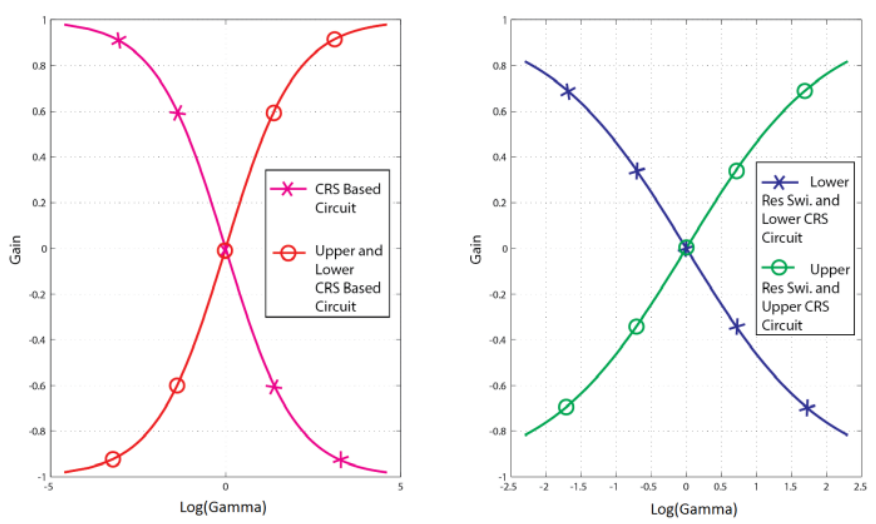

Fig. 12. Gains of the negative-positive gain amplifiers for $\beta=100$.

With both upper and lower CRS's converges to +1 and -1 quicker than the other NPGAs do. All the NPGA's given in Fig. 11 can be used to make perceptrons. In this paper, only the CRS based negative-positive gain amplifier is used in the perceptron and ANN circuit simulations. The CRS based perceptron circuit with the CRS based negative-positive gain amplifier can be seen in Fig. 9. $\beta$ is also an important parameter for CRS based memories [8] and is defined as the ratio of the maximum resistance to the minimum resistance and calculated as

$$
\beta=\frac{R_{O F F}}{R_{O N}}
$$

Eq. (16) can be rewritten using $\beta$ parameter for all of the proposed negative-positive gain amplifiers shown in Fig. 11. The CRS based negative positive gain amplifier gains are derived and put in Table II. The maximum and minimum gain values of the CRS based negative-positive gain amplifier are calculated as

$$
\begin{gathered}
G_{\max }=\frac{R_{O F F}-R_{O N}}{R_{O F F}+R_{O N}}=\frac{\beta-1}{\beta+1} \\
G_{\min }=\frac{R_{O N}-R_{O F F}}{R_{O N}+R_{O F F}}=\frac{1-\beta}{1+\beta}
\end{gathered}
$$




\begin{tabular}{l|l|l|}
$\begin{array}{l}\text { TABLE II MAXIMUM AND MINIMUM GAIN RATES DEPEND ON } \beta . \\
\begin{array}{l}\text { Types of Negative } \\
\text { Positive Gain } \\
\text { amplifiers }\end{array}\end{array}$ & $\begin{array}{l}\text { Maximum Gain } \\
\text { Equations }\end{array}$ & $\begin{array}{l}\text { Minimum Gain } \\
\text { Equations }\end{array}$ \\
\hline $\begin{array}{l}\text { CRS Based } \\
\text { Negative Positive } \\
\text { Gain Amplifier }\end{array}$ & $G_{\max }=\frac{\beta-1}{\beta+1}$ & $G_{\text {min }}=\frac{1-\beta}{1+\beta}$ \\
\hline $\begin{array}{l}\text { Lower Resistive } \\
\text { Switch Negative } \\
\text { Positive Gain } \\
\text { Amplifier }\end{array}$ & $G_{\max }=\frac{\beta-\sqrt{\beta}}{\beta+\sqrt{\beta}}$ & $G_{\min }=\frac{1-\sqrt{\beta}}{1+\sqrt{\beta}}$ \\
\hline $\begin{array}{l}\text { Upper Resistive } \\
\text { Switch Negative } \\
\text { Positive Gain } \\
\text { Amplifier }\end{array}$ & $G_{\max }=\frac{\sqrt{\beta}-1}{\sqrt{\beta}+1}$ & $G_{\min }=\frac{\sqrt{\beta}-\beta}{\sqrt{\beta}+\beta}$ \\
\hline $\begin{array}{l}\text { Lower CRS } \\
\text { Negative Positive } \\
\text { Gain Amplifier }\end{array}$ & $G_{\max }=\frac{\beta-\sqrt{\beta}}{\beta+\sqrt{\beta}}$ & $G_{\min }=\frac{1-\sqrt{\beta}}{1+\sqrt{\beta}}$ \\
\hline $\begin{array}{l}\text { Upper CRS } \\
\text { Negative Positive } \\
\text { Gain Amplifier }\end{array}$ & $G_{\max }=\frac{\sqrt{\beta}-1}{\sqrt{\beta}+1}$ & $G_{\min }=\frac{\sqrt{\beta}-\beta}{\sqrt{\beta}+\beta}$ \\
\hline $\begin{array}{l}\text { Upper And Lower } \\
\text { CRS Negative } \\
\text { Positive Gain } \\
\text { Amplifier }\end{array}$ & $G_{\max }=\frac{\beta-1}{\beta+1}$ & $G_{\min }=\frac{1-\beta}{1+\beta}$ \\
\hline
\end{tabular}
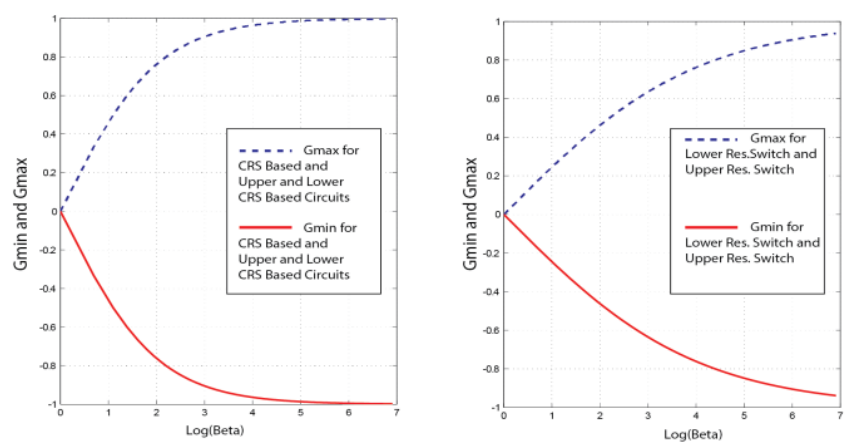

Fig. 13. The maximum and minimum gains of the negativepositive gain amplifiers.

As shown in Fig. 13, two of the six negative positive gain amplifiers have a better convergence than the others according to $\beta$ parameter. The CRS based, upper and lower CRS based negative positive gain amplifiers shows relatively better reaction to $\beta$. Because of this parameter dependency of the gain region, in this paper, it is proposed that the CRS based negativepositive gain amplifier topology must be used. In this study, use of the NPGA with both upper and lower CRSs shown in Fig. 11(f) is also suggested. The threshold voltage of this circuit is equal to almost equal to $2 V_{\boldsymbol{T H}}$ which is twice as high in the other topologies in Fig. 11. Therefore, the programmed ANN input voltage range is higher than the other topologies given in Fig. 11. As mentioned before, the CRS memristance varies if input voltages above the threshold voltage get applied to the perceptron inputs and gains of the NPGA circuits undesirably get destructed. Fig. 11(a),(d) and (e) have also CRSs. However, as it can be seen in hysteresis curves as shown in Fig. 4, one of the resistive switches of the CRSs changes their memristance values when the input voltage exceeds $\boldsymbol{V}_{\boldsymbol{T} \boldsymbol{}} \boldsymbol{1}$ in either direction. The absolute value of the input voltage value required to change the CRS resistance for must be between the threshold voltage values between $V_{T H 1}$ and $V_{T H 2}$ for the topologies given in Fig. 11(a),(d) and (e). The circuit in Fig. 11(f) has two CRSs connected in series and, therefore, also doubles the applicable input voltage. The absolute value of the input voltage value required to change the CRS resistance for the topology given in Fig. 11(f) must be between the threshold voltage values between $2 V_{T H 1}$ and $2 V_{T H 2}$. It should not be forgotten that only the value of one of the resistive switches in the CRS changes its value not the other considering the input voltage and resistive switch polarity.

\section{Activation function}

The activation function of an Adaline perceptron circuit can be obtained using an opamp as a saturator as shown in Fig. 14. The activation function of a perceptron can be given as,

$$
f(u)=\left\{\begin{array}{cc}
1, & u>0 \\
-1, & u \leq 0
\end{array}\right.
$$

The output voltage of the activation function circuit is dependent on the saturation voltage of the opamp:

$$
v_{\text {out }}= \begin{cases}V_{+S A T} & , \quad u>0 \\ V_{-S A T} & , \quad u \leq 0\end{cases}
$$

Where $V_{+S A T}$ is the positive saturation voltage and $V_{-S A T}$ is the negative saturation voltage. If $V_{+S A T}=-V_{-S A T}$, the output voltage of the activation function circuit is

$$
v_{\text {out }}=V_{+S A T}\left\{\begin{array}{cc}
1, & u>0 \\
-1, & u \leq 0
\end{array}\right.
$$

The output of the activation function circuit can be limited by using the anti-series connected zener diodes shown in Fig. 14;

$$
v_{\text {out }}=\left(V_{Z}+V_{T H}\right)\left\{\begin{array}{cc}
1, & u>0 \\
-1, & u \leq 0
\end{array}\right.
$$

Where $V_{Z}$ and $V_{T H}$ are diode threshold and zener diode voltages.

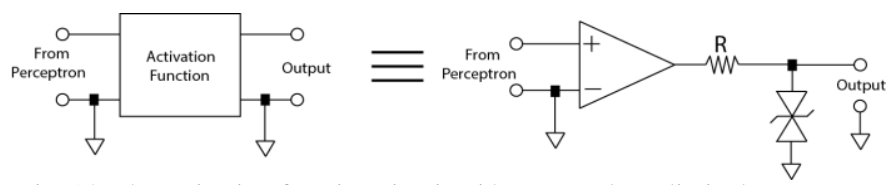

Fig. 14: The activation function circuit with zener voltage limited output. 


\section{A MICROPROCESSOR-BASED PROGRAMMER FOR THE CRS- BASED PERCEPTRON AND/OR ANN CIRCUIT}

In this section, a microcontroller-based programmer circuit is given in Fig. 15. It can be used to program the upper and lower CRS-based perceptron circuits. The programmer circuit is made of also two selector switches. The selective switches can also be made using mosfets or other semiconductor circuit elements. The ADC is used for measuring the voltage across the lower CRS switch. The voltage across the resistor $R_{s}$ is used to limit and measure the lower CRS switch current. The microcontroller uses the selector switches to select the CRS cell desired to be programmed and then gives a negative pulse to bring (to reset) the lower CRS value to $R_{\text {on }}$ and then uses either negative or positive pulses to obtain the memristance value necessary for the desired gains. Using the lower CRS switch current and voltage, microcontroller stops the pulses when the desired memristance value is obtained with an acceptable error in the range of $\left[R_{o n}, R_{o f f}\right]$. The voltage of the $k^{\text {th }} \mathrm{CRS}$ shown in Fig. 15 is

$$
V_{k}=\frac{C R S_{k}\left(q_{k}\right)}{R_{s}+C R S_{k}\left(q_{k}\right)} \cdot V_{p u l s e}
$$

The current of the selected CRS is

$$
i_{m e m(k)}=\frac{V_{\text {pulse }}}{R_{s}+C R S_{k}\left(q_{k}\right)}
$$

Where $\mathrm{CRS}_{\mathrm{k}}$ is memristance of $\mathrm{k}^{\text {th }}$ CRS element and $\mathrm{q}_{\mathrm{k}}$ is charge of $k^{\text {th }}$ CRS element. $R_{s}$ is a resistor which is connected to CRS element serially, $V_{\text {pulse }}$ is impulse voltage level. $V_{k}$ is voltage on $k$.th CRS element, $i_{\text {mem(k) }}$ current of CRS element when element exited with $\mathrm{V}_{\text {pulse }}$ voltage.

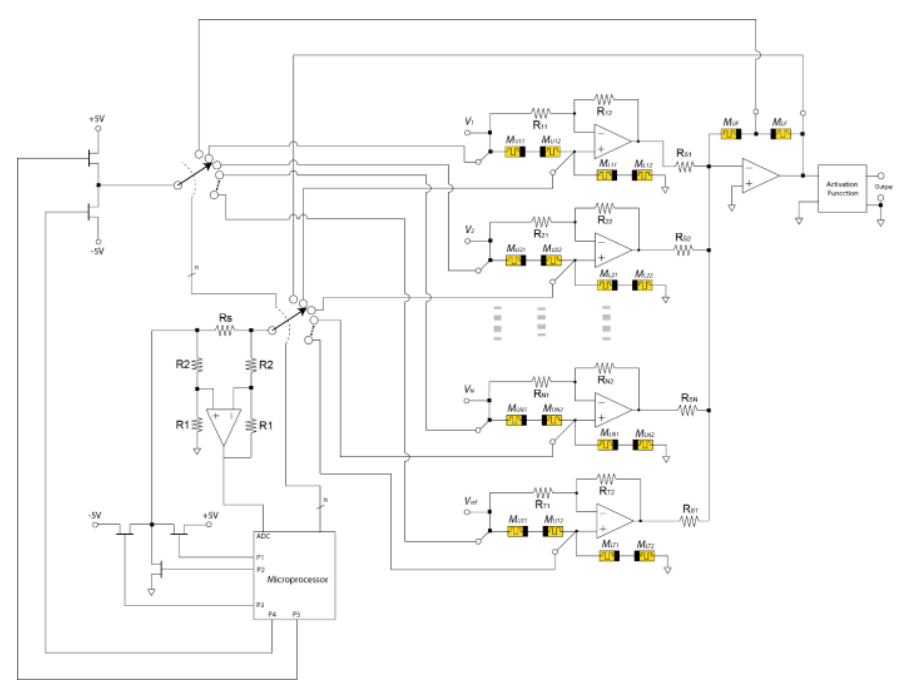

Fig. 15. The Microprocessor based programmer circuit.

\section{A. Programming Waveforms}

By applying consequent pulses, the lower cell resistance of the CRS can be adjusted to the value desired. The resistance modulation waveforms of the lower CRS cell with a pulse train is shown in Fig. 16 and 17.

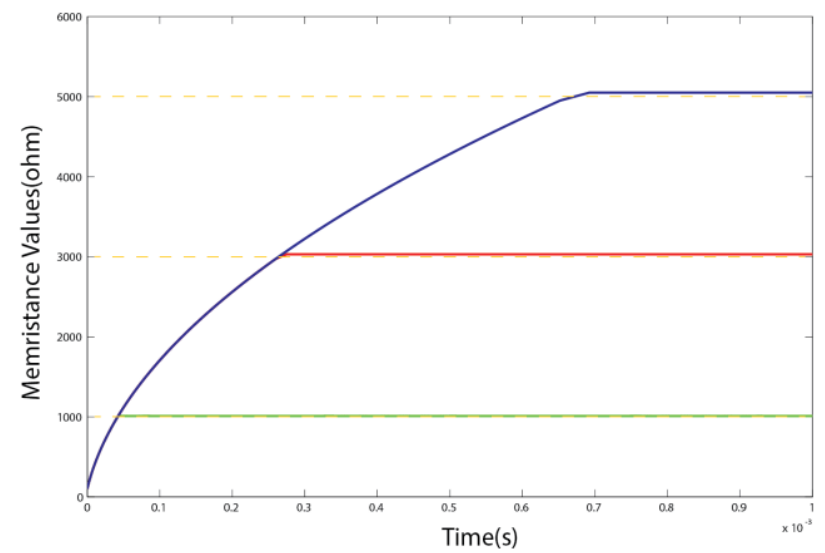

Fig. 15. The lower memristance vs time for the aimed memristance value being $1000 \Omega$ with a $\% 1$ permited memristance error gap.

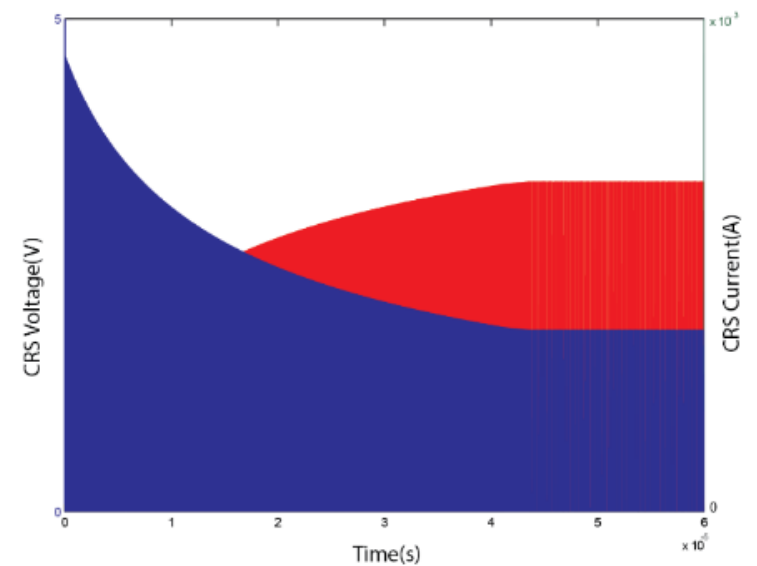

Fig. 16. Memristor voltage and current vs time for the aimed memristance value being $1000 \Omega$ with a $\% 1$ permited memristance error tolerance.

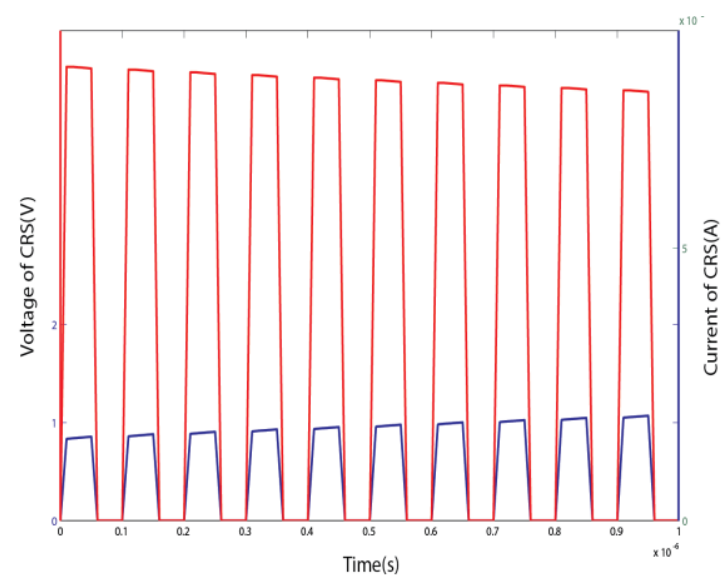

Fig. 17. A zoomed view of a section from Fig. 16. 


\section{Simulation Results of Proposed Perceptron Model}

Perceptron is a linear separator. In this study, two linear separable set inputs are applied to the perceptron and the simulation results are examined to verify its performance. Due to data in the third column, the set is linearly separable. The set $\{\mathrm{X}, \mathrm{Y}\}$ is

$$
\begin{aligned}
& X=\left[\begin{array}{l}
X_{1} \\
X_{2} \\
X_{3} \\
X_{O}
\end{array}\right]=\left[\begin{array}{cccc}
0.9340 & 0.4694 & 0.0119 & 0.3112 \\
0.1656 & 0.6541 & 0.6892 & 0.2290 \\
0.5 & 0.5 & -0.5 & -0.5 \\
1 & 1 & 1 & 1
\end{array}\right] \\
& Y=\left[\begin{array}{llll}
+1 & +1 & -1 & -1
\end{array}\right]
\end{aligned}
$$

Considering also DC offset input, the CRS-based perceptron circuit requires four inputs. The weighting factors obtained from MATLAB simulations which are needed to linearly separate the set is given in eq. (28).

$$
V_{O}=Y=\left[\begin{array}{llll}
+1 & +1 & -1 & -1
\end{array}\right]
$$

The output voltage given in Eq. (29) is the same as the output set given in Eq. (28).

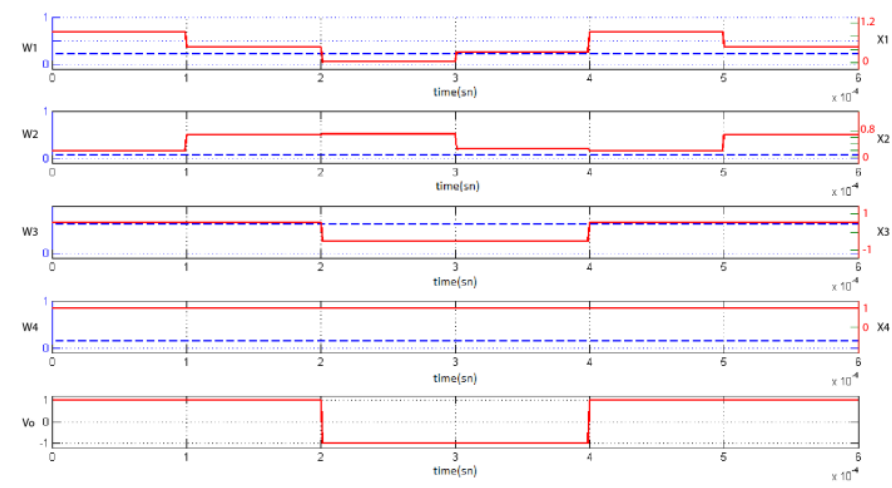

Fig. 18. Inputs and outputs of CRS based perceptron model.

Perceptron simulation results for input voltages higher than the equivalent CRS threshold voltage is given in Fig. 19. The plots drawn with dashed lines shown in the first four rows in Fig. 19 are the gains of the perceptron or the NPGAs for the related inputs. As shown in Fig.19, the perceptron gains belonging to the inputs $X_{1}, X_{3}$ and $X_{4}$ gets destroyed in a short time when higher input voltages than the CRS threshold voltages applied. The gain of $X_{2}$, the second input of the NPGA is destroyed at $0.1 \mathrm{msec}$ since the input voltage exceeds the threshold voltage. As a result, the output voltage plot shows linear separation operation by the perceptron circuit cannot be made by the perceptron due to the fact that its gains have been destroyed with the higher input voltages applied.

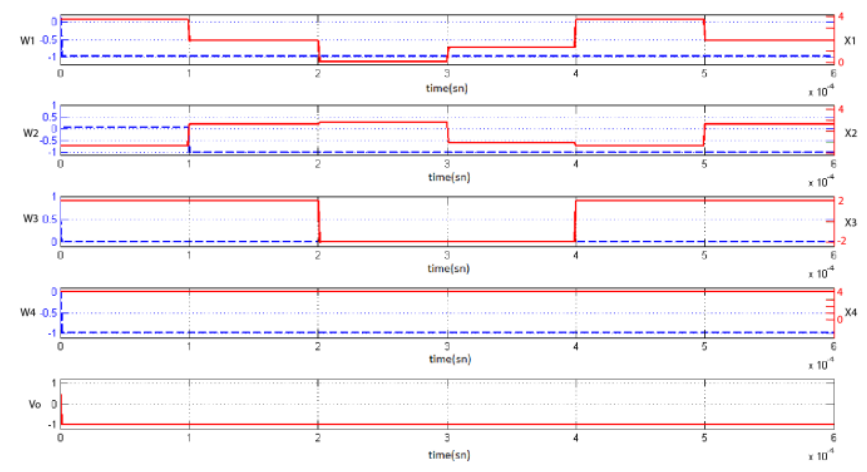

Fig. 19. The CRS-based perceptron simulation results while input voltages exceed the CRS threshold voltage.

\section{DISCUSSIONS}

A CRS-based perceptron and ANN circuits are developed for the first time in the literature. Their operation principles are given. The input voltages applied to the perceptron must be less than the equivalent threshold voltage of the CRS cells and, therefore, is able to keep the memristance values constant. Perhaps, also a scaling circuit may be added to the CRS-based perceptron circuit for future work.

A microntroller-based programmer circuit is also suggested for the CRS-based perceptron and ANN circuits. A method to program them circuit is given. The CRS cells are programmed using the programmer circuit which is able to apply higher negative or positive voltage pulses than the equivalent threshold voltage to the chosen lower cells to bring their memristances to the value which gives the needed feedback gains or the needed weights by the perceptron circuit.

Also, for the first time in the literature, it is shown how to obtain negative and positive gains with a CRS-based opamp circuit which can be used for all analog perceptron and ANN circuits. In the Future, the CRS based perceptron circuit topology may find usage in the market as a cheap and reliable solution for the needed ANN circuits.

\section{REFERENCES}

[1] Dan W. Patterson, Artificial neural networks: theory and applications. Prentice Hall PTR, 1998.

[2] M. Janardan, I. Saha, "Artificial neural networks in hardware: A survey of two decades of progress", Neurocomputing Vol:74, No:1, 2010, pp.239-255.

[3] D. F. Morgado, A. Antunes, A. M. Mota, "Artificial neural networks: a review of commercial hardware", Engineering Applications of Artificial Intelligence, Vol:17, No:8, 2004, pp.945-952.

[4] D.B. Strukov, G.S. Snider, , D.R. Stewart, R.S. Williams, "The missing memristor found", Nature, Vol: 453, 2008, pp. 80-83.

[5] L.O. Chua, "Memristor - the missing circuit element", IEEE Trans Circuit Theory, Vol.18, 1971, pp. 507-519.

[6] T. Prodromakis, C. Toumazou "A Review on Memristive Devices and Applications" Electronics, Circuits, and Systems (ICECS), 17th IEEE International Conference on, 2010, pp. $934-937$. 
[7] L. Chua, "Resistance switching memories are memristors." Applied Physics A, Vol.102, No.4, 2011, pp. 765-783.

[8] E. Linn, R. Rosezin, C. Kügeler, R. Waser, "Complementary resistive switches for passive nanocrossbar memories" Nature Mater., vol.9, 2010, pp. 403-406.

[9] M. A. Zidan, H. H. Fahmy, M. M. Hussain, K.N. Salama, "Memristor-based memory: The sneak paths problem and solutions" Microelectronics Journal, Vol.44, No.2, 2012, pp. 176-183.

[10] R. Rosezin, E. Linn, L. Nielen, C. Kügeler, R. Bruchhaus, R. Waser, "Integrated Complementary Resistive Switches for Passive High-Density Nanocrossbar Arrays", Electron Device Letters, vol. 32, No.2, 2011, pp. 191193.

[11] A. Fabien, E. Zamanidoost, D. B. Strukov. "Pattern classification by memristive crossbar circuits using ex situ and in situ training", Nature communications, Vol. 4, 2013, p.2072.

[12] L. Wang, D. Meitao, D. Shukai, "Memristive perceptron for combinational logic classification", Mathematical Problems in Engineering, 2013.

[13] B. Li, Y. Wang, Y. Wang, Y. Chen, H. Yang, "Training itself: Mixedsignal training acceleration for memristor-based neural network", In Design Automation Conference (ASP-DAC), 2014 19th Asia and South Pacific, 2014, pp. 361-366.

[14] http://www.technologyreview.com/news/537211/a-better-way-to-buildbrain-inspired-chips/

[15] E. Karakulak, R. Mutlu, E. Uçar, "Reconstructive sensing circuit for complementary resistive switches based crossbar memories", Turk J Elec Eng \& Comp Sci, Vol. 24, 2016,pp. 1371-1383.

[16] Y. Yuchao, P. Sheridan, W. Lu, "Complementary resistive switching in tantalum oxide-based resistive memory devices." Applied Physics Letters, Vol.100 No.20, 2012, p.203112.

[17] C. Yang, "Nanoscale bipolar and complementary resistive switching memory based on amorphous carbon", Electron Devices, IEEE Transactions on Vl.58 No.11, 2011, pp. 3933-3939.

[18] J.J. Hopfield, D. W. Tank, "Neural" computation of decisions in optimization problems." Biological cybernetics, Vol.52 No.3, 1985, pp. 141152.

[19] J.J. Hopfield, "Neurons with graded response have collective computational properties like those of two-state neurons." Proceedings of the national academy of sciences, Vol.81, No.10, 1984, pp. 3088-3092.

[20] R. Berdan, T. Prodromakis, C. Toumazou, "High precision analogue memristor state tuning." Electronics letters, Vol. 48, No.18, 2012, pp. 11051107.

[21] W. Yi, F. Perner, M.S. Qureshi, H. Abdalla, M.D. Pickett, J.J. Yang, R.S. Williams, "Feedback write scheme for memristive switching devices", Applied Physics A, Vol. 102, No. 4, pp. 973-982.

\section{BIOGRAPHIES}

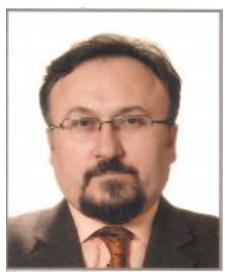

Erdem Uçar was born in Edirne, Turkey in 1966. He received B.Sc. degree from Trakya University in Physiscs in 1990, M.Sc and Ph.D. degrees from Trakya University in Computer Engineering in 1993 and 1996 respectively. $\mathrm{He}$ is currently professor at Management Information Systems Department, Trakya University, Edirne, Turkey. His research interests include management information systems and operating systems.

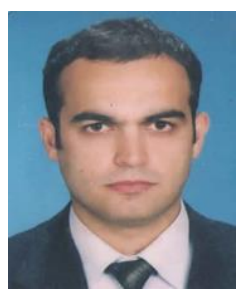

Ertuğrul Karakulak was born in Tekirdağ, Turkey in 1979. He received B.Sc. degree from Sakarya University in Electronics Education in 2001, M.Sc degree in 2005 and Ph.D. degree from Trakya University in 2016. He is currently a assistant professor at Electronics Department, Namik Kemal University, Vocational School of Technical Sciences. His research interests include memristor and resistive RAMs.

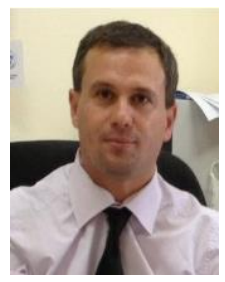

Reşat Mutlu was born in Tekirdağ, Turkey in 1973. He received B.Sc. degree from YILDIZ Teknik University in Electrical Engineering in 1995 and M.Sc. and Ph.D. degree from Rensselaer Polytechnic Institute in Electric Power Engineering in 1998 and 2004, respectively. He is currently associated professor at Electronics and Telecommunication Engineering Department, Namik Kemal University, Tekirdag, Turkey. His main research interests are modeling of memristor and memristive systems, analog and computer memory applications of memristive systems, and modeling of memcapacitive and meminductive systems. 